\title{
High frequency of Taenia solium antigen positivity in patients admitted for neurological disorders in the Rural Hospital of Mosango, Democratic Republic of Congo
}

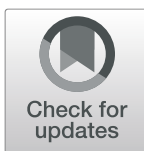

Deby Mukendi ${ }^{1} 2^{2 *}$ (D, Jean-Roger Lilo Kalo ${ }^{1}$, Pascal Lutumba ${ }^{1,2}$, Barbara Barbé ${ }^{3}$, Jan Jacobs ${ }^{3,4}$, Cedric P. Yansouni ${ }^{5}$, Sarah Gabriël ${ }^{6}$, Pierre Dorny ${ }^{7}$, François Chappuis ${ }^{8}$, Marleen Boelaert ${ }^{9}$, Andrea S. Winkler $^{10,11}$, Kristien Verdonck $^{9}$ and Emmanuel Bottieau ${ }^{3}$

\begin{abstract}
Background: The epidemiology of human cysticercosis and neurocysticercosis, caused by the larval stage of the pork tapeworm Taenia solium, is not well known in the Democratic Republic of Congo (DRC). Within a multicenter etiological and diagnostic study conducted by the NIDIAG consortium ("Better Diagnosis for Neglected Infections") and investigating several challenging syndromes, we consecutively evaluated from 2012 to 2015 all patients older than 5 years presenting with neurological disorders (neurology cohort) and with fever $>7$ days (persistent fever cohort) at the rural hospital of Mosango, province of Kwilu, DRC. In both cohorts, etiological diagnosis relied on a systematic set of reference laboratory assays and on pre-established clinical case definitions. No neuroimaging was available in the study hospital. In this study, we determined the frequency of $T$. solium infection in both cohorts and explored in the neurology cohort its association with specific neurological presentations and final etiological diagnoses.
\end{abstract}

Methods: We conducted a post-hoc descriptive and analytic study on cysticercosis in the neurology and persistent fever cohorts, based on the presence in serum samples of circulating T. solium antigen using the B158/B60 enzymelinked immunosorbent assay (ELISA) and of cysticercosis lgG using the LDBIO Cysticercosis Western Blot IgG assay.

\footnotetext{
* Correspondence: debymukendi@gmail.com

'Institut National de Recherche Biomédicale, Kinshasa, Democratic Republic of the Congo

${ }^{2}$ Départment de Neurologie, Université de Kinshasa, Kinshasa, Democratic Republic of the Congo

Full list of author information is available at the end of the article
}

(c) The Author(s). 2021 Open Access This article is licensed under a Creative Commons Attribution 4.0 International License, which permits use, sharing, adaptation, distribution and reproduction in any medium or format, as long as you give appropriate credit to the original author(s) and the source, provide a link to the Creative Commons licence, and indicate if changes were made. The images or other third party material in this article are included in the article's Creative Commons licence, unless indicated otherwise in a credit line to the material. If material is not included in the article's Creative Commons licence and your intended use is not permitted by statutory regulation or exceeds the permitted use, you will need to obtain permission directly from the copyright holder. To view a copy of this licence, visit http://creativecommons.org/licenses/by/4.0/. The Creative Commons Public Domain Dedication waiver (http://creativecommons.org/publicdomain/zero/1.0/) applies to the data made available in this article, unless otherwise stated in a credit line to the data. 


\begin{abstract}
(Continued from previous page)
Results: For the neurology cohort, 340 samples (of 351 enrolled patients) were available for analysis (males: 46.8\%; mean age: 38.9 years). T. solium antigen positivity was found in 43 participants (12.6\%; 95\% confidence interval [Cl] 9.3-16.7\%), including 9 of 60 (15\%) patients with epilepsy. Among the 148 samples available from the persistent fever cohort (males: 39.9\%; mean age: 19.9 years), 7 were positive in the T. solium antigen ELISA (4.7\%; 95\% Cl 1.9$9.5 \% ; P=0.009$ when compared to the neurology cohort). No significant association was found within the neurology cohort between positivity and clinical presentation or final diagnoses. Of note, the lgG antibodydetecting assay was found positive in only four (1.3\%) of the participants of the neurology cohort and in none of the persistent fever cohort.
\end{abstract}

Conclusions: T. solium antigen positivity was found in at least $10 \%$ of patients admitted with neurological disorders in the Kwilu province, DRC, with no specific pattern of presentation. Further neuroimaging studies should be used to confirm whether neurocysticercosis is prevalent in this region.

Keywords: Neurocysticercosis, Taenia solium, Neurology, Serological test, Democratic Republic of Congo, Crosssectional study

\section{Background}

Cysticercosis is an infection caused by the metacestode larval stage of the tapeworm Taenia solium that affects pigs and humans, in areas where sanitation and veterinary control are poor. Pigs act as intermediate hosts by ingesting $T$. solium eggs released in human feces. Humans are the only definitive hosts able to harbor adult tapeworms in the intestines, that may cause a rather mild illness called taeniasis. Humans, however, may also develop cysticercosis after ingestion of eggs, if the food/water or environment is contaminated with human fecal material, or by self-infection in case of adult worm carriage [1]. Larval cysts (also called cysticerci) may settle in different tissues (muscles, skin) but are preferentially localized in the central nervous system (brain and spinal cord), causing neurocysticercosis, a disease associated with major morbidity, poor quality of life and significant cost for the health-care system, particularly in low-resource settings $[2,3]$. Depending on the number, size, stage, and location of the cysticerci as well as the host's immune response, neurocysticercosis may cause a wide variety of neurological symptoms and signs, including seizures, headaches, focal deficits, psychiatric manifestations and cognitive impairment [4-6]. On the other hand, some of the people carrying cysticerci in the central nervous system or in other locations may remain fully asymptomatic [2]. An accurate diagnosis of neurocysticercosis almost always requires a combination of epidemiological, clinical, imaging and serological information, as very few elements are fully diagnostic in isolation. Definitions of probable or definitive diagnosis of neurocysticercosis are based on a set of neuroimaging and clinical/exposure major and minor criteria, which have been recently revised by Del Brutto et al. [7]. Major neuroimaging criteria include cystic lesions, enhancing lesions, multilobulated cysts (all these lesions are usually referred to as active neurocysticercosis) and calcifications (referred to as inactive neurocysticercosis, in the absence of other active lesions). Wellstandardized immunoassays are used as major "clinical/ exposure criteria" and may detect either circulating $T$. solium antigens, reflecting the presence of living cysticerci in any tissue (current/active cysticercosis), or antibodies, which may be present in case of exposure and in current or past infection [8]. For this reason, sensitivity of antigen-based assays is usually lower than that of antibody-based assays for diagnosing cysticercosis [9]. Of note, in low-resource settings, brain imaging and most immunoassays are usually not available or not affordable, making the definitive diagnosis of neurocysticercosis rarely possible $[3,10]$.

Neurological disorders account for approximately $10 \%$ of all admissions in African rural hospitals [11-13]. However, the etiological spectrum has been hardly studied so far because of lack of diagnostic facilities [14]. To fill this knowledge gap, we investigated the infectious etiologies of neurological disorders in the rural hospital of Mosango, province of Kwilu, Democratic Republic of Congo (DRC) [15], as part of the NIDIAG project("Better diagnosis of Neglected Infectious Diseases", https:// nidiag.eu/) that investigated the etiologies of several challenging syndromes in various tropical countries. In this rural DRC setting where neuroimaging is not available, the contribution of neurocysticercosis in the neurological case load is unknown. T. solium is highly endemic in the Bas Congo province, west of the capital Kinshasa, and is suspected to be present in other parts of the country as well [15]. The aim of this study was to determine the frequency of positivity of antigen- and antibody-based T. solium immunoassays in the clinical samples of the participants of the NIDIAG neurology study (neurology cohort), as well as to explore the association with the clinical presentation and final established diagnosis. A secondary objective was to determine 
the frequency of immunoassay positivity in another NIDIAG cohort of patients evaluated for persistent fever ( 7 days) in the same study hospital and during the same period (persistent fever cohort). This cohort of patients with non-neurological symptoms as used as comparator.

\section{Methods}

\section{Study participants and setting}

We conducted a post-hoc descriptive and analytic study for cysticercosis in the serum samples of the participants of the neurology and persistent fever cohorts, based on the presence of $T$. solium antigen and antibodies. The rationale, objective, design, study population and setting of the NIDIAG neurology study have been described in detail previously [15]. Briefly, all consecutive children older than 5 years and adults presenting with neurological disorders to the "Hôpital Général de Référence" (HGR) of Mosango, province of Kwilu were prospectively enrolled and evaluated. Entry criteria included any of the following: altered state of consciousness; change in sleep pattern; cognitive decline; changes in personality/behavior; recent ( $<2$ weeks) epileptic seizure; recent, severe and progressive headache; meningism; new onset cranial nerve lesion; new onset sensory-motor focal deficit; and new onset gait/walking disorders. Participants underwent a systematic diagnostic workup including a microscopic examination of cerebrospinal fluid (CSF) unless this was contra-indicated. Neuro-imaging diagnostic methods were not available at the study site. A pre-established set of severe and treatable infections of the central nervous system (CNS) were considered as "targeted infections" for the NIDIAG study. They comprised human African trypanosomiasis, malaria, bacterial meningitis, unspecified meningo-encephalitis, HIVrelated neurological disorders, CNS tuberculosis and neurosyphilis. These targeted infections were searched for systematically with reference methods (either on site, or in reference laboratories in Kinshasa, DRC or Antwerp, Belgium). Other diagnoses (of non-targeted conditions) were made in 2016 by a panel of experts in neurology and infectious diseases, who reviewed the complete clinical case files and all laboratory results and followed pre-established case definitions [15]. Three hundred fifty-one patients were enrolled in the NIDIAG neurology study between 2012 and 2015 .

In parallel, from 2013 to 2015, 300 children $>5$ years and adults presenting to the same study hospital with persistent fever (defined as fever reported or documented for more than 7 days) were prospectively included in the NIDIAG persistent fever study. They also underwent a systematic diagnostic work-up to explore a pre-established specific set of targeted severe and treatable etiologies.

\section{Samples and laboratory procedures}

All sera of the NIDIAG studies were shipped on dry ice to the Institute of Tropical Medicine, Antwerp and stored in the biobank at $-80^{\circ} \mathrm{C}$. For the present study, we selected all available serum samples from the entire NIDIAG neurology cohort and a subset of 150 samples from the persistent fever cohort (every other consecutive participant). In April 2018, these serum samples were processed for the detection of circulating cysticercal antigens and antibodies. The presence of circulating cysticercal antigen was measured in the serum using the in house B158/B60 enzyme-linked immunosorbent assay (Ag-ELISA). The optical density (OD) for each sample was divided by a cut-off OD value to obtain a ratio. The sample was considered positive for cysticercal antigens if the ratio was greater than $1.0[16,17]$. At this cut-off, the assay has a sensitivity of 90 to $100 \%$ to detect current cysticercosis and a specificity of 83 to $98 \%$ [10, 18]. To determine the presence of antibodies against $T$. solium infection, a commercial Western Blot assay using lentillectin purified glycoprotein antigens was used (Cysticercosis WB IgG, LDBIO Diagnostics, Lyon, France). This assay is considered to provide information on exposure to/current and past infection with $T$. solium, with a sensitivity of $98 \%$ and a specificity of $100 \%$ according to the manufacturer [19]. A result is considered positive if a minimum of two of the five bands react with the serum sample in the Western Blot. The laboratory technician who performed both $T$. solium assays was blinded to all clinical and diagnostic information. When the available serum volume was small, the antigen-detecting assay was prioritized.

\section{Data analysis and reporting}

Data were analyzed with SPSS software, version 25.0 (SPSS, Chicago, IL, USA). For both the NIDIAG neurology and persistent fever cohorts, the frequency of $T$. solium positivity was determined with a $95 \%$ confidence interval $(\mathrm{CI})$ and stratified by age group. Within the NIDIAG neurology cohort, the association of T. solium antigen positivity with clinical features and final diagnosis was also assessed. Baseline epidemiological characteristics and frequency data were compared between cohorts. Chi-square test or Fisher's exact test were used for categorical variables. Continuous variables were compared using parametric tests. All tests were two-sided and $p$ values $<0.05$ were considered statistically significant.

\section{Ethical aspects}

The study protocol was approved by the Institutional Review Board of the Institute of Tropical Medicine, Antwerp, Belgium and by the Ethics Committees of the University of Antwerp, Belgium (reference 11/50/400; 2012), 
and the Public Health School of Kinshasa, DRC (reference ESP/CE/016/2012). The study was registered at clinicaltrials.gov (identifier NCT01589289). Before enrolment, participants or their legal guardians gave written informed consent for study participation and for the future use of their stored clinical samples for further etiological or diagnostic research. For children between 12 and 18 years, in addition to the parental consent, informed assent was necessary for inclusion, in line with the DRC law.

\section{Results}

Serum samples were available for 340 out of the 351 participants (97\%) of the NIDIAG neurology cohort (Table 1). The mean age of this study population was 38.9 years [standard deviation: 17.7; range: $6-78$ ); and the male-to-female ratio was $0.88(159 / 181)$. Duration of neurological symptoms was more than 2 weeks in 190 (55.9\%) of the evaluated patients and almost half had prior contact with a primary health care facility. Severe headache was the most frequent complaint, followed by gait/walking disorders and seizures. Confirmed targeted infections accounted for 84 (24.7\%) of the final diagnoses while 156 (45.9\%) cases were classified as noncommunicable conditions, including mainly epilepsy and psychiatric disorders (Table 1). Death occurred in 26 patients (7.6\%). The antigen-detecting assay was positive in $43 / 340$ (12.6\%; 95\% CI 9.3-16.7\%) participants of the NIDIAG neurology cohort. The antibody-detecting assay could be done in 314 samples, and was positive in four of them (1.3\%; 95\% CI 0.5-3.2\%). All four antibodypositive cases were also T. solium antigen positive.

Table 1 Baseline features, final diagnoses and outcome of 340 patients of the NIDIAG neurology cohort with available T. solium antigen results

\begin{tabular}{|c|c|c|c|c|}
\hline & $\begin{array}{l}\text { Total participants evaluated } \\
(n=340)\end{array}$ & $\begin{array}{l}\text { TS antigen negative participants } \\
(n=297)\end{array}$ & $\begin{array}{l}\text { TS antigen positive participants } \\
(n=43)\end{array}$ & $P$ \\
\hline Features at presentation & n (\%) & n (\%) & n (\%) & \\
\hline Male sex & $159(46.8)$ & $137(46.1)$ & $22(51.2)$ & 0.5 \\
\hline Female sex & $181(53.2)$ & $160(53.8)$ & $21(48.8)$ & 0.5 \\
\hline Age $>20$ years & $270(79.4)$ & $231(77.8)$ & $39(90.7)$ & 0.05 \\
\hline $\begin{array}{l}\text { Prior contact with primary care } \\
\text { facility }\end{array}$ & $159(46.8)$ & $141(47.5)$ & $18(41.9)$ & 0.4 \\
\hline $\begin{array}{l}\text { Neurological symptoms > two } \\
\text { weeks }\end{array}$ & $190(55.9)$ & $168(56.6)$ & $22(51.2)$ & 0.5 \\
\hline Fever reported/documented & $99(29.1)$ & $89(30.0)$ & $10(23.3)$ & 0.3 \\
\hline Severe headache & $156(45.9)$ & $136(45.8)$ & $20(46.5)$ & 0.9 \\
\hline Severe headache without fever & $101(29.7)$ & $86(29.0)$ & $15(34.9)$ & 0.4 \\
\hline Gait/walking disorders & $97(28.5)$ & $86(29.0)$ & $11(25.6)$ & 0.6 \\
\hline Seizure & $84(24.7)$ & $73(24.6)$ & $11(25.6)$ & 0.8 \\
\hline Focal sensory-motor deficit & $77(22.6)$ & $67(22.6)$ & $10(23.3)$ & 0.9 \\
\hline $\begin{array}{l}\text { Cognitive and/or behavior } \\
\text { disturbance }\end{array}$ & $72(21.2)$ & $60(20.2)$ & $12(27.9)$ & 0.2 \\
\hline Altered state of consciousness & $69(20.3)$ & $61(20.5)$ & $8(18.6)$ & 0.7 \\
\hline Final diagnoses & n (\%) & n (\%) & n (\%) & \\
\hline Confirmed targeted infections & $84(24.7)$ & $76(25.6)$ & $8(18.6)$ & 0.3 \\
\hline $\begin{array}{l}\text { Confirmed and suspected } \\
\text { infections }\end{array}$ & $119(35.0)$ & $107(36.0)$ & $12(27.9)$ & 0.2 \\
\hline Non-communicable conditions & $156(45.9)$ & $134(45.1)$ & $22(51.2)$ & 0.4 \\
\hline Epilepsy & $60(17.6)$ & $51(17.2)$ & $9(20.9)$ & 0.5 \\
\hline Psychiatric disorders & $53(15.6)$ & $47(15.8)$ & $6(14.0)$ & 0.7 \\
\hline $\begin{array}{l}\text { Myelo-radiculo-neuropathic } \\
\text { syndromes }\end{array}$ & $37(10.9)$ & $30(10.1)$ & $7(16.3)$ & 0.2 \\
\hline Cerebrovascular accident & $23(6.8)$ & $20(6.7)$ & $3(7.0)$ & 0.9 \\
\hline Outcome & n (\%) & n (\%) & n (\%) & \\
\hline Death & $26(7.6)$ & $23(7.7)$ & $3(7.0)$ & 0.8 \\
\hline
\end{tabular}

TS denotes Taenia solium 
Within the neurology cohort, there were no differences regarding presenting features and final grouped or single diagnoses between $T$. solium antigen-positive and -negative participants (Table 1). There was only a trend for higher frequency of patients older than 20 years in the $T$. solium antigen-positive group $(P=0.05)$.

In the neurology cohort, the frequency of $T$. solium antigen positivity was $12.6 \%$ in patients with reported/ documented fever and $13.7 \%$ in patients without fever $(P=0.2)$. Slight non-significant variations in frequency were observed between the presenting neurological symptoms in the whole cohort or when restricted to non-febrile patients. T. solium antigen was positive in $8 /$ 84 (9.5\%) of patients with confirmed targeted infection and in 22/156 (14\%) of those finally diagnosed with noncommunicable conditions, but this difference was not statistically significant $(P=0.3)$. Antigen positivity was observed in 9 of 60 (15\%) patients diagnosed with epilepsy.

Of the 150 samples obtained from the patients with persistent fever, 148 could be analyzed with both antigen- and antibody-based tests. The male-to-female ratio was lower than in the neurology cohort $(0.66 ; 59 / 89)$, but this difference was not statistically significant (Table 2). In contrast, mean age was 19.9 years (standard deviation: 16.2; range: 6-72) in this cohort, and was significantly lower than that of the neurology cohort $(p<$ $0.001)$. T. solium antigen was positive in $7 / 148$ (4.7\%; 95\% CI 1.9-9.5\%). No single case of positive T. solium antibody assay was found. As shown in Table 2, the frequency of $T$. solium antigen positivity was significantly higher in the neurology compared to the persistent fever cohort $(P=0.009$; odds ratio 2.9 ; $95 \%$ confidence interval 1.3-6.6). When we stratified by age, the frequency of positivity remained higher in the neurology cohort, but was only statistically significant for subjects over 40 years of age.

\section{Discussion}

In this post-hoc analysis of a prospective cohort of patients presenting with neurological disorders in a rural hospital of Central Africa, we found that about 13\% had evidence of circulating $T$. solium antigen in serum. There were no clear associations between presenting symptoms or final diagnoses and positivity for T. solium antigen. The frequency of antigen positivity was significantly higher in the neurology cohort than in the "comparator" persistent fever cohort (approximatively 5\%), but a statistical difference was only observed in the subgroup of participants older than 40 years. Surprisingly, the antibody-based immunoassay was positive in only $1.3 \%$ of the neurological patients and in none of the persistent fever cohort.

This exploratory study has many limitations, most of which were largely acknowledged in previous publications reporting on the NIDIAG findings in DRC $[15,20$, $21]$. The source NIDIAG study was not initially designed to investigate risk factors for neurocysticercosis since the presence of this disease had not been reported in humans in the region so far. The use of patients rather than a sample of the general population is another limitation of this post-hoc analysis as it does not enable the full magnitude of the underlying problem to be estimated. A complete diagnostic workup was restricted to a set of targeted infections for both the neurology and the persistent fever studies. In particular for the neurology study, the etiological workup was limited by the absence of advanced neurological investigations in this low-resource hospital. Consequently, no causative link can be formally established between the serological

Table 2 Epidemiological features and frequency of $T$. solium antigen and antibody positivity in the neurology and persistent fever cohorts evaluated at the rural hospital of Mosango, Democratic Republic of Congo

\begin{tabular}{|c|c|c|c|}
\hline & Neurological cohort $(n=340)$ & Persistent fever cohort $(n=148)$ & $P$ \\
\hline \multicolumn{4}{|l|}{ Epidemiological data } \\
\hline Male sex, n (\%) & $159(47)$ & $59(40)$ & 0.1 \\
\hline Female sex, n (\%) & $181(53)$ & $89(60)$ & 0.1 \\
\hline Mean age (SD), years & $39.9(17.7)$ & $19.9(16.2)$ & $<0.001$ \\
\hline \multicolumn{4}{|l|}{ Positive T. solium antigen assay } \\
\hline Total group, n (\%) & $43(12.6)$ & $7(4.7)$ & 0.009 \\
\hline Age group $\leq 20$ years, $\mathrm{n}(\%)$ & $4(1.1)$ & $3(2)$ & 0.4 \\
\hline Age group $21-40$ years, $n\left(\%^{\circ}\right.$ & $17(5)$ & $3(2)$ & 0.1 \\
\hline Age group > 40 years, n (\%) & $22(6.4)$ & $1(0.7)$ & 0.006 \\
\hline \multicolumn{4}{|l|}{ Positive T. solium antibody assay } \\
\hline Total group n/n (\%) & 4/314 (1.3) & 0/148 (0) & 0.3 \\
\hline
\end{tabular}

Note: $S D$ denotes standard deviation 
markers of T. solium infection and the neurological presentation in the absence of brain/spinal imaging that could demonstrate cysticerci. Also, the persistent fever cohort was opportunistically used for comparison, but cannot be considered as a "control" group as such since the distribution of age groups was different. Finally, we acknowledge that the surprisingly low prevalence of $T$. solium antibodies in the neurology cohort may be considered an important weakness. Indeed, one would have expected a proportion of antibody-positive participants, indicating exposure to T. solium eggs, larger than that of antigen positivity. A possible explanation is that some degradation of the T. solium immunoglobulins has inflated the false negative rate when the antibody assay was performed in 2018, while blood sampling took place between 2012 and 2015. However, transport and storage were rigorously monitored during the study and other serological investigations on the same stored samples did provide plausible results [22]. Alternatively, this may have been related to differences in the circulating strains of $T$ solium, parasitic load or host characteristics compared to settings where the antibody-detection assay was validated [23]. A failure of the LDBIO assay itself is unlikely since it performed well with positive and negative controls. However, sensitivity of this commercial assay has been reported in one study as substantially inferior to that of the reference Centers of Disease Controldeveloped enzyme-linked electroimmunotransfer blot (CDC-EITB), i.e. $13.3 \%$ versus $52.2 \%$ among 23 CTconfirmed neurocysticercosis cases tested [24]. On the other hand, in a recent Mexican study on 58 NCC patients, the sensitivity of the LDBIO EITB was slightly higher (71.4\%) than that of the CDC-EITB (66.1\%) [25]. These discrepancies between reference EITB test sensitivities need further investigation on a larger number of samples. In general, other types of commercial antibodybased-assays such as ELISA have limited sensitivities and specificities for diagnosing neurocysticercosis [26].

The African continent reports the highest burden of human cysticercosis in the world, with pooled prevalence of circulating $T$. solium antigens estimated at $7.30 \%$ (95\% CI $4.23-12.31)$ and of antibodies at $17.37 \%$ (95\%CI 3.33-56.20) in community-based epidemiological surveys [25]. In DRC, the epidemiology of human and porcine cysticercosis is poorly understood. Some surveys in the animal markets of Kinshasa have revealed active cysticercosis in pigs originating from different DRC provinces [18], including in more than $30 \%$ of those coming from Bandundu (the former name of the Kwilu province). Very high prevalence of human cysticercosis (proportion of T. solium antigen positivity up to $21 \%$ ) has been reported in the only communitybased survey performed so far, in the province of Bas Congo [16].
It is generally accepted that a higher proportion of neurocysticercosis cases is usually found in the older age categories [27, 28]. We found some similar trend when looking at $T$. solium antigen positivity, but cannot strongly support this observation since age categories were not evenly distributed in both cohorts and we did not specifically investigate neurocysticercosis. In contrast, we found no evidence of a difference in gender prevalence in our study. This agrees with findings reported in Bolivia [29], but contrasts with data from Guatemala and Tanzania where a higher prevalence was found in females [30,31], and with findings in DRC and Vietnam, which showed a higher prevalence in males $[32,33]$. These differences might be explained by different living conditions and the distribution of household tasks between genders in those countries.

The prevalence of neurocysticercosis remains unknown in most low-resource settings, since neuroimaging is required to establish the diagnosis [34]. The frequency of probable and definitive neurocysticercosis has been mainly investigated in patients with epilepsy, in whom the pooled estimate obtained from brain CT Scan-based studies was 30\%, globally [34] and in Latin America [29]. Additional studies performed later on in Africa revealed that the proportion of neurocysticercosis in patients with epilepsy varied a lot according to the setting and could range from 15 to $50 \%$ [24, 31, 32].

Diagnostic accuracy studies of antigen- and antibodybased assays for the specific diagnosis of neurocysticercosis (based on CT findings) in Africa have been limited to small case series. Using Del Brutto's definition of probable or definitive neurocysticercosis in patients evaluated for epilepsy in Zambia, Gabriël et al. found a sensitivity of $44 \%$ (15 positive results/34 cases) for $T$. solium antigen, when using a ratio of 1.0 as the cutoff, and a specificity of $90 \%(10)$. When restricting the analysis to patients with active neurocysticercosis (i.e. presence of viable cystic lesions, $n=6$ ), the sensitivity was $100 \%$ and the specificity $84 \%$. Antibody-based Western Blot assays are usually reported as highly sensitive for neurocysticercosis in patients with more than one viable cystic lesion, but performance is much lower in cases of single lesions or of calcifications only. The specificity of antibodybased tests for neurocysticercosis is variable since distinction with previous exposure or cured infection cannot be made [23]. As already mentioned, diagnostic performance of antibody-based ELISA assays is even poorer [26].

Based on all these considerations, the sizeable proportion of participants with positive $T$. solium antigen in both cohorts strongly suggests that active cysticercosis and neurocysticercosis are endemic in the study area, since false-positive results are infrequent for both 
conditions. Also, the high frequency of antigen positivity in patients with neurological disorders is compatible with the hypothesis that this infection plays some causal role $[4,32]$. It is worth reminding in addition that neurocysticercosis tends to become clinically apparent when the cystic lesions degenerate, through local inflammatory reactions, while active cysts alone usually cause little neurological symptoms [35]. This may partly explain its age-dependent distribution.

Neuroimaging is absolutely necessary not only to accurately diagnose neurocysticercosis, but also to safely manage the potential risk of clinical deterioration due to anti-helminthic treatment (albendazole and/or praziquantel). While proven useful for epidemiological surveys and under study for assessing public health interventions (such as mass drug administration with anti-helminthic drugs in endemic regions), the utility of immunoassays is more questionable for clinical care, since no therapeutic decision can exclusively rely on their results [23, 35]. Antigen- or antibody-based assays, whenever available at the point-of-care [36, 37], could however be explored as an operational screening tool to select the subset of neurological patients who might benefit most from neuroimaging [38]. Since such investigations would be difficult to obtain and expensive in rural Congo, choosing assays or cutoffs with high specificity to diagnose active neurocysticercosis (the main form responsive to anti-helminthic treatment) needs to be prioritized, in order to minimize the number of unnecessary referrals. Prior to such a study however, adequate treatment of neurocysticercosis has to be made available in African low-resource hospitals [3].

\section{Conclusion}

In this retrospective analysis of hospital-based neurology and persistent fever cohorts in the Kwilu province of DRC, T. solium antigen positivity was frequent (about 13 and $5 \%$, respectively), confirming that this infection is prevalent in the region. Although the causal link with neurocysticercosis could not be formally established, our findings suggest that $T$. solium infection may contribute to the neurological case load in Central African areas. Further research should aim at performing brain imaging in neurological patients found with circulating antigens to fully characterize the spectrum of $T$. soliumassociated morbidity in rural DRC, and at identifying risk factors for T. solium infection in order to design appropriate control measures targeting both the final and intermediate hosts.

\section{Abbreviations}

CNS: Central Nervous System; CSF: Cerebrospinal fluid; DRC: Democratic republic of Congo, The; ELISA: Enzyme-Linked Immunosorbent assay; HGR: Hôpital Général de Référence; NIDIAG: Neglected Infectious diseases DIAGnosis; OD: Optical Density

\section{Acknowledgements}

We would like to thank all medical and nursing staff of the general referral hospital of Mosango, as well as all study participants.

\section{Authors' contributions}

Designed research: EB, MB, FC, JJ, PL, CY, ASW. Enrolled patients, performed and registered clinical diagnosis: DM, EB, CY, ASW. Performed laboratory experiments: JRLK, BB, PD, SG. Analyzed the data: EB, KV, PD, SG. Wrote the paper: EB, DM, MB, JJ, PD, SG, FC, CY, ASW, KV. Agree with manuscript results and conclusions: all authors. The author(s) read and approved the final manuscript.

\section{Funding}

This work is part of the NIDIAG European research network (Collaborative Project), supported by the European Union's Seventh Framework Programme for research, technological development, and demonstration under grant agreement no. 260260. The funders had no role in study design, data collection, and analysis, decision to publish, or preparation of the manuscript. CPY holds a "Chercheur-boursier clinicien" career award from the Fonds de recherche du Québec - Santé (FRQS).

\section{Availability of data and materials}

The Institute of Tropical Medicine of Antwerp, Belgium (sponsor of this study) is exploring the public repositories that allow making all material and data available with sufficient security. Meanwhile, the dataset is available on request.

\section{Declarations}

Ethics approval and consent to participate

As mentioned under materials and Methods, ethics approvals were obtained from the Institutional Review Board of the Institute of Tropical medicine and the Ethical Committee of the University Hospital, Antwerp, Belgium as well as the School of Public Health of the University of Kinshasa, DR Congo. All study participants signed an informed consent before inclusion in this studies and accepted that additional investigations could be performed later on retrospectively on their samples for etiological purposes.

\section{Consent for publication}

Not applicable.

\section{Competing interests}

The authors report no conflict of interest.

\section{Author details \\ 'Institut National de Recherche Biomédicale, Kinshasa, Democratic Republic of the Congo. 'Départment de Neurologie, Université de Kinshasa, Kinshasa, Democratic Republic of the Congo. ${ }^{3}$ Department of Clinical Sciences, Institute of Tropical Medicine, Antwerp, Belgium. ${ }^{4}$ Department of Microbiology and Immunology, KU Leuven, Leuven, Belgium. ${ }^{5}$ JD MacLean Centre for Tropical Diseases, McGill University Health Centre, Montreal, Canada. ${ }^{6}$ Department of Veterinary Public Health, Faculty of Veterinary Medicine, Ghent University, Ghent, Belgium. 'Department of Biomedical Sciences, Institute of Tropical Medicine, Antwerp, Belgium. ${ }^{8}$ Division of Tropical and Humanitarian Medicine, Geneva University Hospitals and University of Geneva, Geneva, Switzerland. ${ }^{9}$ Department of Public Health, Institute of Tropical Medicine, Antwerp, Belgium. ${ }^{10} \mathrm{Center}$ for Global Health, Department of Neurology, Technical University of Munich, Munich, Germany. \\ ${ }^{11}$ Center for Global Health, University of Oslo, Oslo, Norway.}

Received: 15 December 2019 Accepted: 5 April 2021

Published online: 17 April 2021

\section{References}

1. García HH, Gonzalez AE, Evans CA, Gilman RH, Cysticercosis Working Group in Peru. Taenia solium cysticercosis. Lancet. 2003;362(9383):547-56. https:// doi.org/10.1016/S0140-6736(03)14117-7.

2. Garcia HH. Neurocysticercosis. Neurol Clin. 2018;36(4):851-64. https://doi. org/10.1016/j.ncl.2018.07.003. 
3. Winkler AS. Neurocysticercosis in sub-Saharan Africa: a review of prevalence, clinical characteristics, diagnosis, and management. Pathog Glob Health. 2012;106(5):261-74. https://doi.org/10.1179/2047773212Y.0000000047.

4. Carabin $\mathrm{H}$, Ndimubanzi PC, Budke CM, Nguyen H, Qian Y, Cowan LD, et al. Clinical manifestations associated with neurocysticercosis: a systematic review. PLoS Negl Trop Dis. 2011;5(5):e1152.

5. Wallin MT, Pretell EJ, Bustos JA, Caballero M, Alfaro M, Kane R, et al. Cognitive changes and quality of life in neurocysticercosis: a longitudinal study. PLoS Negl Trop Dis. 2012;6(1):e1493.

6. Nau AL, Mwape KE, Wiefek J, Schmidt K, Abatih E, Dorny P, et al. Cognitive impairment and quality of life of people with epilepsy and neurocysticercosis in Zambia. Epilepsy Behav. 2018;80:354-9. https://doi. org/10.1016/j.yebeh.2017.10.042.

7. Del Brutto OH, Nash TE, White AC, Rajshekhar V, Wilkins PP, Singh G, et al. Revised diagnostic criteria for neurocysticercosis. J Neurol Sci. 2017;372:20210. https://doi.org/10.1016/j.jns.2016.11.045.

8. Praet N, Rodriguez-Hidalgo R, Speybroeck N, Ahounou S, Benitez-Ortiz W, Berkvens D, et al. Infection with versus exposure to Taenia solium: what do serological test results tell us? Am J Trop Med Hyg. 2010;83(2):413-5. https://doi.org/10.4269/ajtmh.2010.10-0121.

9. Zoli AP, Nguekam N, Shey-Njila O, Nsame Nforninwe D, Speybroeck N, Ito A, et al. Neurocysticercosis and epilepsy in Cameroon. Trans R Soc Trop Med Hyg. 2003;97(6):683-6. https://doi.org/10.1016/S0035-9203(03)80103-7.

10. Gabriël S, Blocher J, Dorny P, Abatih EN, Schmutzhard E, Ombay M, et al. Added value of antigen ELISA in the diagnosis of neurocysticercosis in resource poor settings. PLoS Negl Trop Dis. 2012;6(10):1-8.

11. Bower JH, Asmera J, Zebenigus M, Sandroni P, Bower SM, Zenebe G. The burden of inpatient neurologic disease in two Ethiopian hospitals. Neurology. 2007;68(5):338-42. https://doi.org/10.1212/01.wnl.0000252801. 61190.e8.

12. Philip-Ephraim EE, Eyong Kl, Chinenye S, William UE, Ephraim RP. The burden of inpatient neurologic disease in a tropical African hospital. Can J Neurol Sci. 2013;40(4):576-9. https://doi.org/10.1017/S0317167100014694.

13. Mosser P, Schmutzhard E, Winkler AS. The pattern of epileptic seizures in rural Tanzania. J Neurol Sci. 2007;258(1-2):33-8. https://doi.org/10.1016/j. jns.2007.02.015.

14. Yansouni $C P$, Bottieau $E$, Lutumba $P$, Winkler $A S$, Yansouni $C P$, Bottieau $E$, et al. Rapid diagnostic tests for neurological infections in central Africa. Lancet Infect Dis. 2013;13:546-58.

15. Mukendi D, Lilo Kalo J-RJ-R, Mpanya A, Minikulu L, Kayembe T, Lutumba P, et al. Clinical Spectrum, etiology, and outcome of neurological disorders in the rural Hospital of Mosango, the Democratic Republic of Congo. Am J Trop Med Hyg. 2017;97(5):1454-60. https://doi.org/10.4269/ajtmh.17-0375.

16. Kanobana K, Praet N, Kabwe C, Dorny P, Lukanu P, Madinga J, et al. High prevalence of Taenia solium cysticerosis in a village community of BasCongo, Democratic Republic of Congo. Int J Parasitol. 2011;41(10):1015-8. https://doi.org/10.1016/j.jpara.2011.06.004.

17. Dorny P, Phiri IK, Vercruysse J, Gabriel S, Willingham AL, Brandt J, et al. A Bayesian approach for estimating values for prevalence and diagnostic test characteristics of porcine cysticercosis. Int J Parasitol. 2004;34(5):569-76. https://doi.org/10.1016/j.ijpara.2003.11.014.

18. Praet N, Kanobana K, Kabwe C, Maketa V, Lukanu P, Lutumba P, et al. Taenia solium cysticercosis in the democratic Republic of Congo: how does pork trade affect the transmission of the parasite? PLoS Negl Trop Dis. 2010;4(9): e817.

19. Tsang VCW, Brand JA, Boyer AE. An enzyme-linked Immunoelectrotransfer blot assay and glycoprotein antigens for diagnosing human cysticercosis (Taenia Solium). J Infect Dis. 1989;159(1):50-9. https://doi.org/10.1093/ infdis/159.1.50.

20. Boelaert M, Mukendi D, Bottieau E, Kalo Lilo JR, Verdonck K, Minikulu L, et al. A phase III diagnostic accuracy study of a rapid diagnostic test for diagnosis of second-stage human African trypanosomiasis in the Democratic Republic of the Congo. EBioMedicine. 2017;27:11-7.

21. Mukendi D, Bottieau E, Boelaert M, Gillet P, Winkler AS, Jacobs J, et al. Where there is no brain imaging: safety and diagnostic value of lumbar puncture in patients with neurological disorders in a rural hospital of Central Africa. J Neurol Sci. 2018;393(April):72-9. https://doi.org/10.1016/j. jns.2018.08.004.

22. Bottieau E, Mukendi D, Kalo J-RL, Lutumba P, Barbé B, Ramadan K, et al. Potential usefulness of $C$-reactive protein and procalcitonin determination in patients admitted for neurological disorders in rural Democratic Republic of Congo. Sci Rep. 2019;9(1):1-9.

23. Garcia HH, O'Neal SE, Noh J, Handali S, Gilman RH, Gonzalez AE, et al. Laboratory diagnosis of Neurocysticercosis (Taenia solium). J Clin Microbiol. 2018;56(9):e00424-18.

24. Blocher J, Schmutzhard E, Wilkins PP, Gupton PN, Schaffert M, Auer H, et al. A cross-sectional study of people with epilepsy and neurocysticercosis in Tanzania: clinical characteristics and diagnostic approaches. PLoS Negl Trop Dis. 2011;5(6):e1185

25. Hernández M, Astudillo OG, Diego G, De-la-Rosa-Arana JL, Meza-Lucas A, García-Rodea R, et al. Immunodiagnosis of human neurocysticercosis: comparative performance of serum diagnostic tests in Mexico. Parasitol Res. 2019;118(10):2891-9. https://doi.org/10.1007/s00436-019-06425-4.

26. Garcia HH, Castillo Y, Gonzales I, Bustos JA, Saavedra H, Jacob L, et al. Low sensitivity and frequent cross-reactions in commercially available antibody detection ELISA assays for Taenia solium cysticercosis. Trop Med Int Health. 2018;23(1):101-5. https://doi.org/10.1111/tmi.13010.

27. Fleury A. Development of point-of-care tests for Taenia solium: one of the ways to obtain a better diagnosis and therapeutic management of patients, and to reach eradication. Pathog Glob Health. 2019;113:323-4 Taylor and Francis Ltd.

28. Sáenz B, Ruíz-Garcia M, Jiménez E, Hernández-Aguilar J, Suastegui R, Larralde C, et al. Neurocysticercosis: clinical, radiologic, and inflammatory differences between children and adults. Pediatr Infect Dis J. 2006;25(9): 801-3. https://doi.org/10.1097/01.inf.0000233548.81204.97.

29. Carrique-Mas J, lihoshi N, Widdowson MA, Roca Y, Morales G, Quiroga J, et al. An epidemiological study of Taenia solium cysticercosis in a rural population in the Bolivian Chaco. Acta Trop. 2001;80(3):229-35. https://doi. org/10.1016/S0001-706X(01)00161-9.

30. Garcia-Noval J, Allan JC, Fletes C, Moreno E, De Mata F, Torres-Alvarez R, et al. Epidemiology of Taenia solium taeniasis and cysticercosis in two rural Guatemalan communities. Am J Trop Med Hyg. 1996;55(3):282-9. https:// doi.org/10.4269/ajtmh.1996.55.282.

31. Mwanjali G, Kihamia C, Kakoko DVC, Lekule F, Ngowi H, Johansen MV, et al. Prevalence and risk factors associated with human taenia solium infections in Mbozi District, Mbeya Region, Tanzania. PLoS Negl Trop Dis. 2013;7(3): e2102.

32. Musoke A, Dorny P, Kabwe C, Kirezi K, Lukanu K, Lutumba P, et al. Cysticercosis in the Democratic Republic of Congo. Onderstepoort J Vet Res. 2012;79:1 AOSIS (pty) Ltd.

33. Willingham AL 3rd, De NV, Doanh NQ, Cong le D, Dung TV, Dorny P, et al. Current status of cysticercosis in Vietnam. Southeast Asian J Trop Med Public Heal. 2003;34(Suppl 1):35-50.

34. Coral-Almeida M, Gabriël S, Abatih EN, Praet N, Benitez W, Dorny P. Taenia solium human cysticercosis: a systematic review of sero-epidemological data from endemic zones around the world. PLoS Negl Trop Dis. 2015;9(7): e0003919.

35. Ndimubanzi $P C$, Carabin $\mathrm{H}$, Budke $\mathrm{CM}$, Nguyen $\mathrm{H}$, Qian $\mathrm{YJ}$, Rainwater $\mathrm{E}_{\text {, et al }}$ A systematic review of the frequency of neurocyticercosis with a focus on people with epilepsy. PLoS Negl Trop Dis. 2010;4(11):e870.

36. Bruno E, Bartoloni A, Zammarchi L, Strohmeyer M, Bartalesi F, Bustos J, et al. Epilepsy and neurocysticercosis in Latin America: a systematic review and meta-analysis. PLoS Negl Trop Dis. 2013;7(10):e2480.

37. Winkler AS, Blocher J, Auer H, Gotwald T, Matuja W, Schmutzhard E. Epilepsy and neurocysticercosis in rural Tanzania - an imaging study. Epilepsia. 2009; 50(5):987-93. https://doi.org/10.1111/j.1528-1167.2008.01867.x.

38. Garcia HH, Nash TE, Del Brutto $\mathrm{OH}$. Clinical symptoms, diagnosis, and treatment of neurocysticercosis. Lancet Neurol. 2014;13(12):1202-15. https:// doi.org/10.1016/S1474-4422(14)70094-8.

\section{Publisher's Note}

Springer Nature remains neutral with regard to jurisdictional claims in published maps and institutional affiliations. 